\title{
Ataxia, intellectual disability, and ocular apraxia with cerebellar cysts: a new disease?
}

\author{
Poretti, A ; Häusler, Martin ; von Moers, A ; Baumgartner, B
}

\begin{abstract}
Cerebellar cysts are rare findings in pediatric neuroimaging and rather characteristic for dystroglycanopathies and GPR56-related encephalopathy. We aim to report on seven children with cerebellar cysts showing absence of weakness and ruling out mutations within eight dystroglycanopathy genes and GPR56. Data about neurological and ophthalmological features, outcome, and creatine kinase values were collected from clinical histories and follow-up examinations. All MR images were qualitatively evaluated for infra- and supratentorial abnormalities. A SNP 6.0-Array was performed in three children. The POMT1, POMT2, POMGnT1, FKRP, FKTN, LARGE, ISPD, B3GALNT2, and GPR56 genes were screened in all patients by Sanger sequencing. Seven children from five families were studied. Ataxia, intellectual disability, and language impairment were found in all patients, ocular motor apraxia in five, and severe myopia in three. None of the patients had weakness, only three a minimally increased creatine kinase value. Qualitative neuroimaging evaluation showed cerebellar cysts and dysplasia in the cerebellar hemispheres and vermis in all children. Additional findings were an enlarged fourth ventricle in all children, vermian hypoplasia and brain stem morphological abnormalities in five. The SNP array showed no pathogenetic imbalances in all children evaluated. In all patients, no mutations were found in POMT1, POMT2, POMGnT1, FKRP, FKTN, LARGE, ISPD, B3GALNT2, and GPR56. The peculiar combination of the same clinical and neuroimaging findings in our patients highly suggests that this phenotype may represent a novel entity, possibly falling within the spectrum of dystroglycanopathies.
\end{abstract}

DOI: https://doi.org/10.1007/s12311-013-0521-8

Posted at the Zurich Open Repository and Archive, University of Zurich

ZORA URL: https://doi.org/10.5167/uzh-105195

Journal Article

Published Version

Originally published at:

Poretti, A; Häusler, Martin; von Moers, A; Baumgartner, B (2014). Ataxia, intellectual disability, and ocular apraxia with cerebellar cysts: a new disease? Cerebellum, 12(1):79-88.

DOI: https://doi.org/10.1007/s12311-013-0521-8 


\title{
Ataxia, Intellectual Disability, and Ocular Apraxia with Cerebellar Cysts: A New Disease?
}

\author{
Andrea Poretti • Martin Häusler • Arpad von Moers • Bastian Baumgartner • \\ Klaus Zerres • Andrea Klein • Chiara Aiello • Francesca Moro • Ginevra Zanni • \\ Filippo M. Santorelli • Thierry A. G. M. Huisman • Joachim Weis • Enza Maria Valente • \\ Enrico Bertini • Eugen Boltshauser
}

Published online: 7 September 2013

(C) Springer Science+Business Media New York 2013

\begin{abstract}
Cerebellar cysts are rare findings in pediatric neuroimaging and rather characteristic for dystroglycanopathies and GPR56-related encephalopathy. We aim to report on seven children with cerebellar cysts showing absence of weakness and ruling out mutations within eight dystroglycanopathy genes and GPR56. Data about neurological and ophthalmological features, outcome, and creatine kinase values were collected from clinical histories and follow-up examinations. All MR images were qualitatively evaluated for infra- and supratentorial abnormalities. A SNP 6.0-Array was performed in three children. The POMT1, POMT2, POMGnT1, FKRP, FKTN, LARGE, ISPD, $B 3 G A L N T 2$, and GPR56 genes were screened in all patients by Sanger sequencing. Seven children from five families were studied. Ataxia, intellectual disability, and language impairment were found in all patients, ocular motor apraxia in five, and severe myopia in three. None of the patients had weakness, only
\end{abstract}

A. Poretti $\cdot$ A. Klein $\cdot$ E. Boltshauser $(\bowtie)$

Department of Pediatric Neurology, University Children's Hospital

of Zurich, Steinwiesstrasse 75, 8032 Zurich, Switzerland

e-mail: Eugen.Boltshauser@kispi.uzh.ch

\section{A. Poretti • T. A. G. M. Huisman}

Section of Pediatric Neuroradiology, Division of Pediatric

Radiology, Russell H. Morgan Department of Radiology and

Radiological Science, The Johns Hopkins University School of

Medicine, Baltimore, MD, USA

\section{Häusler}

Department of Pediatrics, University Hospital RWTH, Aachen, Germany

\author{
A. von Moers \\ Department of Pediatrics, DRK Kliniken Berlin Westend, Berlin, \\ Germany \\ B. Baumgartner \\ Pediatric Neurology, Children's Hospital, Landshut, Germany
}

three a minimally increased creatine kinase value. Qualitative neuroimaging evaluation showed cerebellar cysts and dysplasia in the cerebellar hemispheres and vermis in all children. Additional findings were an enlarged fourth ventricle in all children, vermian hypoplasia and brain stem morphological abnormalities in five. The SNP array showed no pathogenetic imbalances in all children evaluated. In all patients, no mutations were found in POMT1, POMT2, POMGnT1, FKRP, FKTN, $L A R G E$, ISPD, B3GALNT2, and GPR56. The peculiar combination of the same clinical and neuroimaging findings in our patients highly suggests that this phenotype may represent a novel entity, possibly falling within the spectrum of dystroglycanopathies.

Keywords Cerebellum · Cerebellar cysts · Neuroimaging · Congenital muscular dystrophy $\cdot$ Dystroglycanopathy

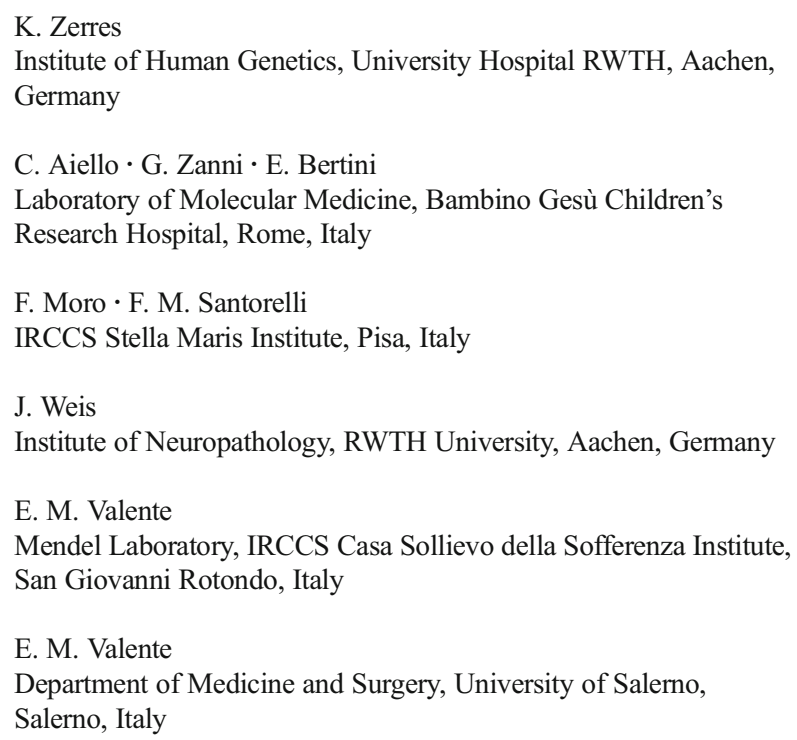




\section{Introduction}

Cerebellar cysts are rare findings in pediatric neuroimaging [1]. They are a rather specific imaging finding for congenital muscular dystrophies (CMDs) because of defective glycosylation of $\alpha$-dystroglycan [2].

CMDs are clinically and genetically heterogeneous with a variable involvement of brain, eyes, and muscles [3, 4]. At the most severe end of the spectrum are Walker-Warburg syndrome (WWS), muscle-eye-brain (MEB) disease, and Fukuyama CMD (FCMD). These syndromes are characterized by muscle weakness, hypotonia and in some cases contractures at birth or within the first 6 months of life, brain malformations (e.g., cobblestone brain as well as cerebellar and brain stem anomalies) resulting in impaired cognitive and motor development, and seizures and eye involvement (e.g., retinal dysplasia and microophthalmia) [2-6]. At the mildest end of the phenotypic spectrum, however, affected patients may present first in adult life with limb-girdle distribution of their muscle weakness without associated brain or eye involvement [7].

To date, mutations in 15 genes involved in glycosylation of $\alpha$-dystroglycan have been associated with CMDs [4, 5, 8-11]. Mutations in these genes account for about half of the patients [3-5]. Additionally, mutations within the GPR56 gene may result in a cobblestone-like lissencephaly with cerebellar cysts [12].

Here, we report on neurological and ophthalmological features, neurodevelopmental outcome, neuroimaging findings, and results of molecular genetic analysis of seven children with a distinctive phenotype characterized by ataxia, intellectual disability, ocular motor apraxia, cerebellar cysts, and cerebellar dysplasia. We hypothesize that these children may represent a new disease, possibly a new phenotype within the dystroglycanopathy spectrum.

\section{Patients and Methods}

This retrospective study did not require approval by the Institutional Review Board. Informed consent for molecular genetics analyses was obtained from the parents of all children included.

\section{Patient Cohort}

The patients included in this study were collected by the senior investigator (EBo) from three sources: (1) his personal database of patients with cerebellar cysts, (2) data of patients with cerebellar cysts referred to EBo for second opinion, and (3) collaboration with other clinical professionals who contributed cases.
The inclusion criterion for this retrospective study was the presence of cysts within the cerebellum on MR images. Exclusion criteria were (1) the presence of cerebellar cysts in children with the diagnosis of well-defined $\alpha$-dystroglycanopathies (clinical and neuroimaging diagnosis with genetic confirmation) and (2) other defined disorders associated with cerebellar cysts including Aicardi syndrome (clinical and neuroimaging findings) $[13,14]$ and pontocerebellar hypoplasia type 6 (clinical and neuroimaging findings) [15].

\section{Clinical Analysis}

Review of the clinical histories and clinical-neurological follow-up examinations provided detailed information about neurological and ophthalmological features as well as neurodevelopmental outcome. Whenever possible, the cognitive and language outcome was evaluated by a formal neuropsychological testing with assessment of the intelligence quotient (IQ) or a standardized developmental testing with assessment of the developmental quotient (DQ). Otherwise, the developmental stage was estimated from the patient's history, clinical observations, and kindergarten or school reports. These details are listed in Table 1.

\section{Qualitative Neuroimaging Analysis}

In a retrospective analysis, two pediatric neurologists with experience in neuroimaging of the pediatric cerebellum (AP and EBo) and a pediatric neuroradiologist (TAGMH) qualitatively analyzed all available neuroimaging data sets. The parameters chosen for evaluation are listed in Table 2. Cerebellar dysplasia was defined as an abnormal cerebellar foliation, fissuration and white matter arborization. The brain stem was assessed on sagittal images according to Doherty et al [16].

\section{Molecular Genetic Analysis}

Genetic analyses included (1) molecular genotyping by using single nucleotide polymorphism (SNP) array with an Affymetrix GeneChip ${ }^{\circledR}$ Genome-Wide Human SNP 6.0-Array (patients 1, 3, and 7; this analysis was not available for the remaining patients) and (2) direct mutation analysis of the coding exons and flanking sequencing of the following genes (all patients): POMT1 (GenBank accession number NM_007171.3), POMT2 (NM_013382.4), POMGnT1 (NM_017739.2), FKRP (NM_001039885.1), FKTN (NM_006731.2), LARGE (NM_004737.3), ISPD (NM_001101426.3), B3GALNT2 (NM_152490.3), and GPR56 (NM_005682).

For mutation analysis, genomic DNA was prepared from peripheral blood samples from patients and available family members according to standard protocols. Sequencing of coding regions and flanking intronic sequences for all analyzed genes 


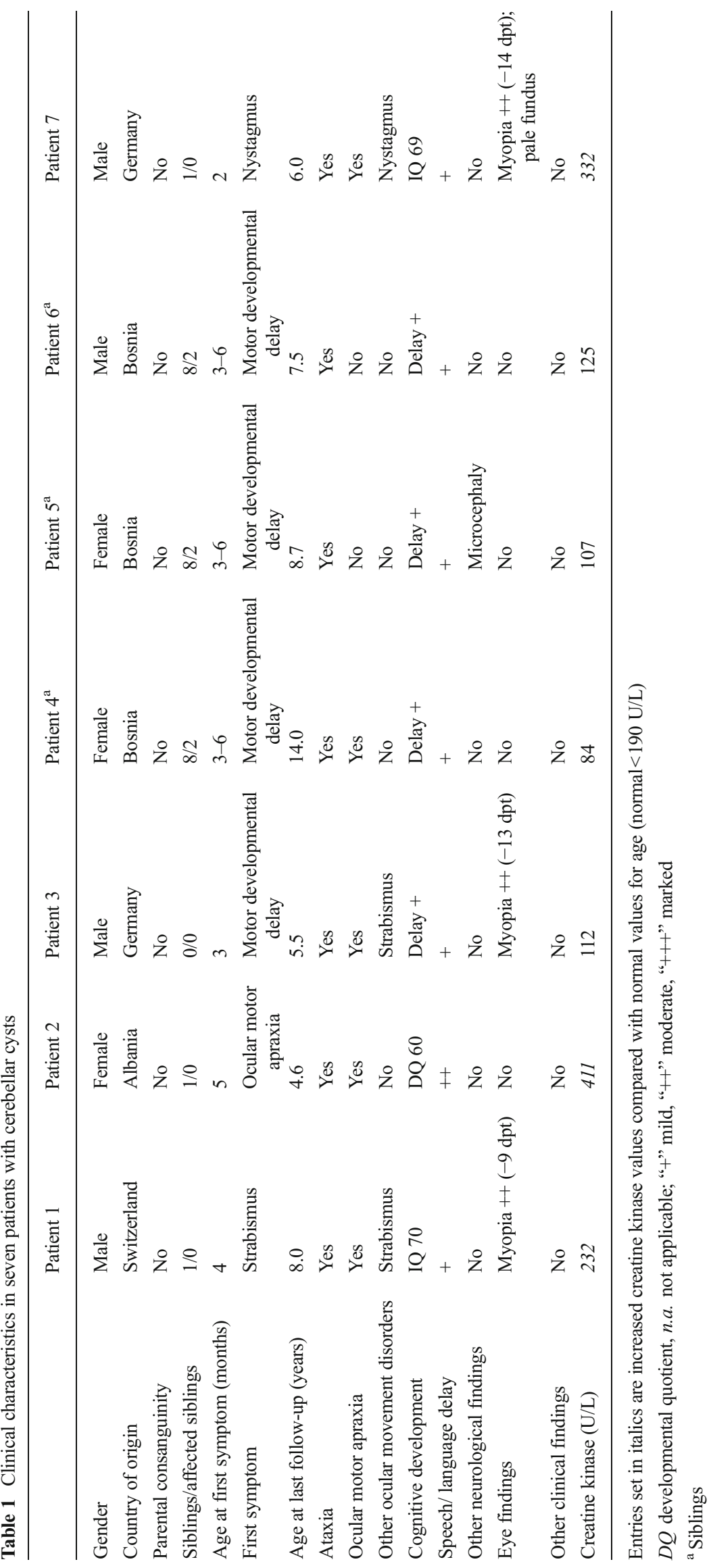




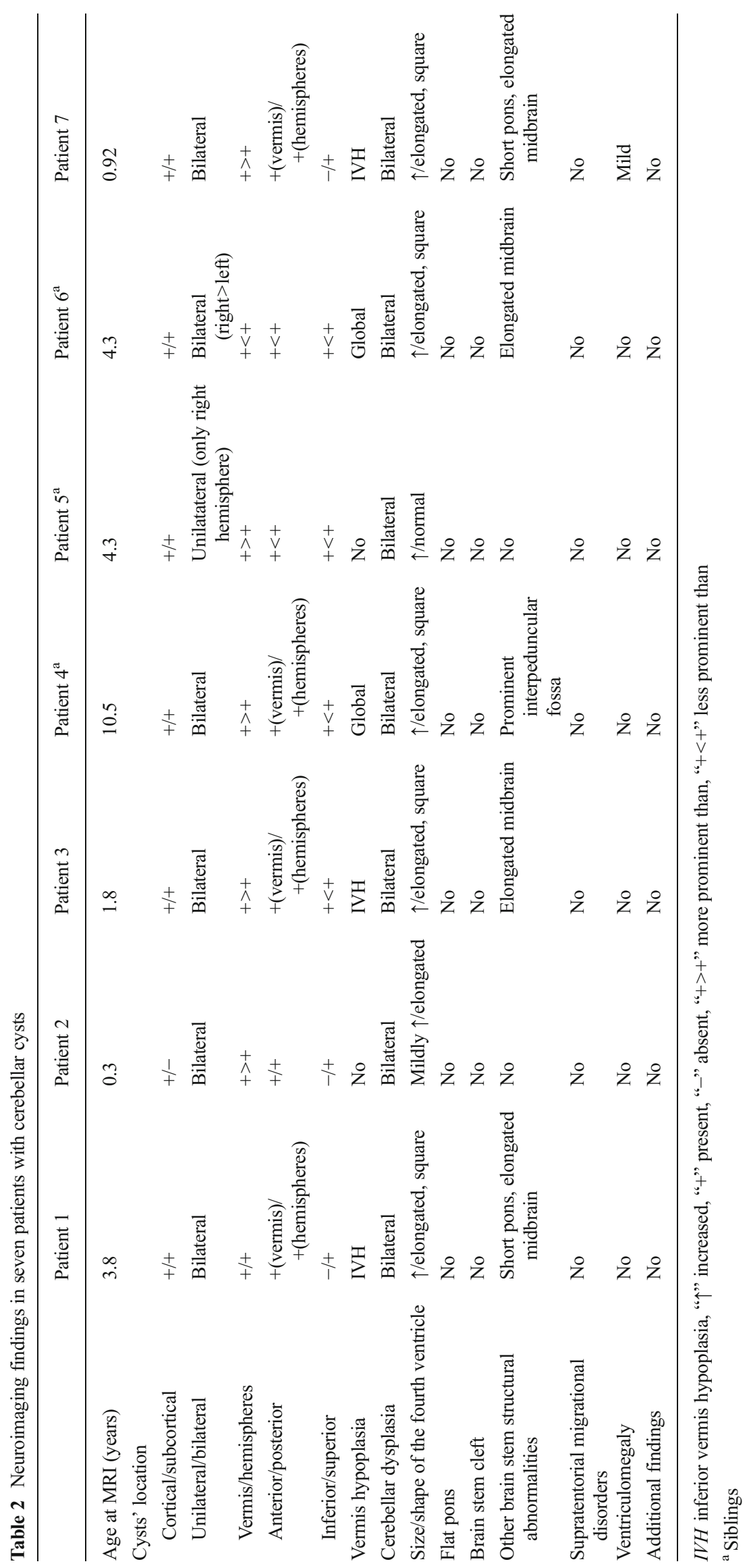


was performed on PCR products after amplification of genomic DNA using oligonucleotide primers and PCR conditions available upon request, and cycle-sequencing adopting the BigDye 3.1 chemistry (Applied Biosystems, Foster City, CA) and following the consensus guidelines for mutation nomenclature (www.hgvs.org/mutnomen). All possible new variants were searched for in a large set of in-house control chromosomes as well as in dbSNP database (http://www.ncbi.nlm.nih.gov/ projects/SNP/) and Exome Variant databases (http://evs.gs. washington.edu/EVS/; https://genomics.med.miami.edu/gvd-hd/ dataSearch.php). New variations were also systematically evaluated in silico to predict their effects on protein function with Condel (http://bg.upf.edu/condel/), Polyphen analysis (http://genetics.bwh.harvard.edu/pph2/index.shtml), and Mutation Taster (http://www.mutationtaster.org/).

\section{Results}

\section{Patient Characteristics and Clinical Findings}

Seven patients (four males and three females) from five unrelated families who showed cerebellar cysts on neuroimaging and fulfilled the above mentioned inclusion criteria were included in this study. At the last follow-up, the median age was 7.5 years (mean, 7.8 years; range, 4.0 to 14.0 years). Pregnancy was uneventful in all children. Patient 7 was born preterm at 36 weeks of gestation. His delivery and perinatal history were uneventful.

The most prominent clinical findings were ataxia $(n=7)$, intellectual disability $(n=7)$, delayed speech/language development $(n=7)$, and ocular motor apraxia (OMA; $n=5)$. The creatine kinase (CK) was elevated in three children. Albumin has been measured in two children and was normal in both. $\alpha$ fetoprotein and cholesterol have been measured in one child and were both normal. More detailed neurological and ophthalmological characteristics are summarized in Table 1.

The cognitive functions of patient 1 were assessed by the non-verbal Snijders-Oomen Nonverbal Intelligence Test (SON-R 2.5-7) at the age of four years. His IQ was 70. The cognitive functions of patient 3 were assessed by the third edition of the Wechsler Preschool and Primary Scale of Intelligence (WPPSI) at the age of six years. His IQ was 69. If available, detailed data on the motor and/or cognitive development are reported for all patients without a standardized cognitive/developmental test. At the age of 4.6 years, the DQ of patient 2 was estimated to be about 60 . She spoke less than ten words. Finally, patient 7 started to walk independently at the age of 3.5 years.

\section{Qualitative Neuroimaging Findings}

All children showed cortical-subcortical cerebellar cysts (bilateral, six of seven) affecting the vermis and the hemispheres as well as bilateral cerebellar dysplasia. Supratentorial migrational disorders and white matter signal abnormalities, by contrast, were not recorded. More detailed results of the qualitative neuroimaging analysis of each patient's MRI (median age of 3.8 years; mean, 3.7 years; range, 3 months to 10.5 years) are summarized in Table 2 . Representative images are shown in Figs. 1, 2, 3, 4, and 5.

\section{Molecular Genetics Findings}

Molecular genotyping by SNP array did not show pathogenetic imbalances in all children evaluated (patients 1, 3, and 7). Variants of disease significance could not be detected in the POMT1, POMT2, POMGnT1, FKRP, FKTN, LARGE, $I S P D, B 3 G A L N T 2$, and GPR56 coding exons and flanking intronic sequences in all patients.

\section{Discussion}

We report on seven children with the same clinical and imaging phenotype characterized by (1) brain involvement as a cerebellar syndrome with nonprogressive ataxia, OMA, and intellectual disability and (2) cerebellar cysts and cerebellar dysplasia. To the best of our knowledge, this peculiar combination of clinical and neuroimaging findings has not been previously reported and may represent a novel syndromic entity. The affection of three siblings (two females and one male) suggests a genetic disorder with autosomal recessive inheritance.

\section{Clinical Phenotype}

The clinical presentation in our patients is characterized by a cerebellar syndrome including nonprogressive ataxia, OMA, and intellectual disability. Intellectual disability and nonprogressive cerebellar ataxia are non-specific features of several diseases [17-19]. The differential diagnosis of OMA is, however, shorter [20]. OMA and nonprogressive cerebellar ataxia typical clinical features of Joubert syndrome [21] and congenital OMA-type Cogan [22]. Additionally, they have been rarely reported in other brain malformations such as agenesis of the corpus callosum and brain stem dysgenesis [23]. These disorders can be easily excluded in our patients because (1) the characteristic neuroimaging findings such as the molar tooth sign in Joubert syndrome and a hypoplastic or normal cerebellum in congenital OMA type Cogan are missing and (2) cerebellar cysts have been never reported in these disorders [23, 24]. Other disorders associated with OMA such as ataxia teleangiectasia and ataxia with oculomotor apraxia types 1 and 2 can be easily excluded in our patients because of the non-progressive course and the neuroimaging findings (cerebellar cysts and dysplasia vs. cerebellar atrophy) [25]. 
Fig. 1 Midsagittal (a), axial (b, c), and coronal (d) T2-weighted MR images of patient 1 at the age of 3.8 years show multiple cortical/subcortical cysts located within the cerebellar vermis (anterior and superior part) and both cerebellar hemispheres (posterior and superior parts). Additional abnormalities illustrated: hypoplasia of the inferior part of the cerebellar vermis (a), bilateral cerebellar dysplasia (b-d), an enlarged fourth ventricle with a peculiar elongated and squared shape (a), an elongated midbrain (a), and a short pons (a)
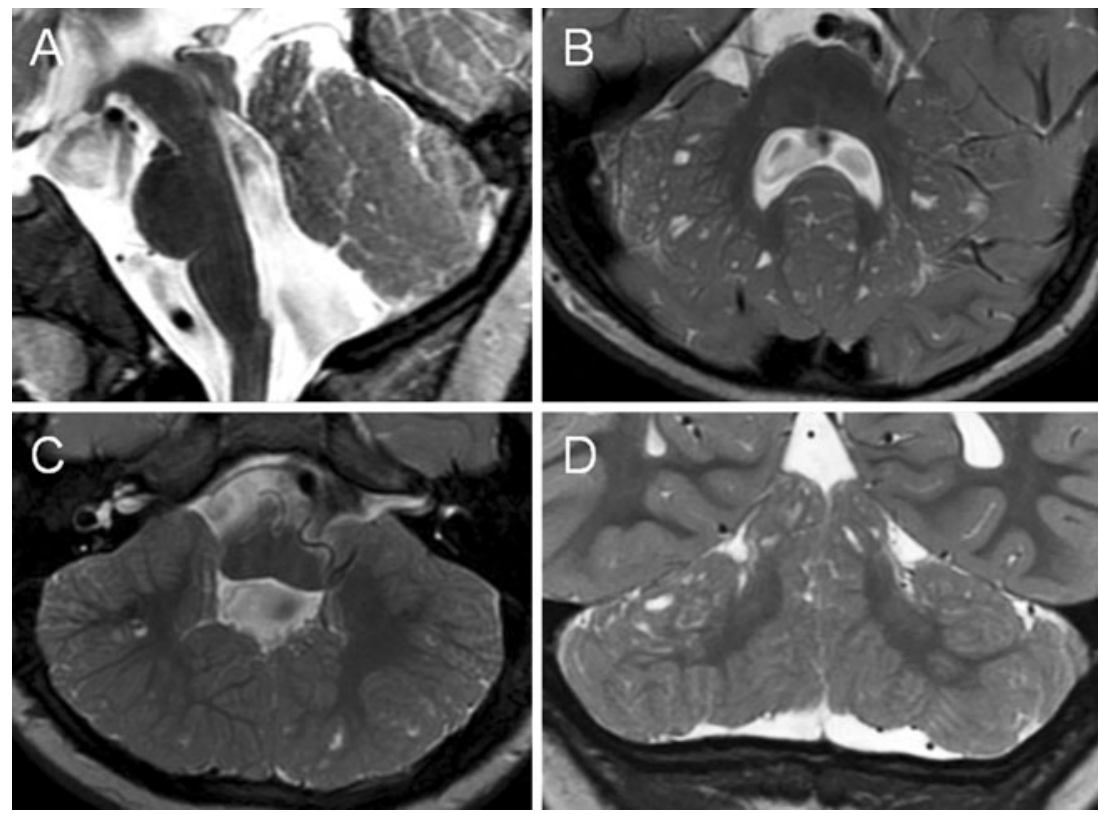

Recently, OMA has been reported in an 8.5-year-old, fully ambulant male with proximal muscle weakness, severe myopia, cerebellar cysts, a $\mathrm{CK}$ value of $1,500 \mathrm{U} / \mathrm{L}$ and mutations in ISPD [26]. To our knowledge, this is the only dystroglycanopathy patient with OMA reported so far. The phenotype of $\alpha$-dystroglycanopathies includes characteristically clinical and laboratory evidence of muscle involvement. Muscle involvement presents as weakness with prenatal or postnatal onset, affects predominantly the proximal muscles and may cause a variable degree of motor impairment ranging from walking with support to almost absent motor development [3]. Hypotonia, contractures and scoliosis are additional frequent features in children with dystroglycanopathy, as are increased $\mathrm{CK}$ values (commonly, $>7$-fold higher than control values) $[3,5]$. The peculiarity of our series of patients is the absence of clinical signs of muscle weakness. Therefore, a muscle biopsy did not appear indicated, although three children showed a slight CK elevation (not higher than 2fold normal values). In dystroglycanopathies, muscle involvement may be isolated or associated with concomitant affection of the central nervous system (CNS) and eyes [3]. CNS and eye involvement are variable and range from mild intellectual disability to absent motor and cognitive development, microcephaly and severe seizures and from myopia, juvenile cataracts to microphthalmia, buphthalmus, and retinal dysplasia, respectively [3]. All our patients had mild to moderate intellectual disability and delayed language development. Three children also had a severe myopia.

Nonprogressive cerebellar ataxia and cerebellar cysts are common features of GPR56-related encephalopathy [12, 27]. To our knowledge, however, OMA has never been reported in GPR56-related encephalopathy $[12,27]$. A predominant cerebellar syndrome with OMA, absence of muscle weakness, and only mildly increased CK value is very unusual and has
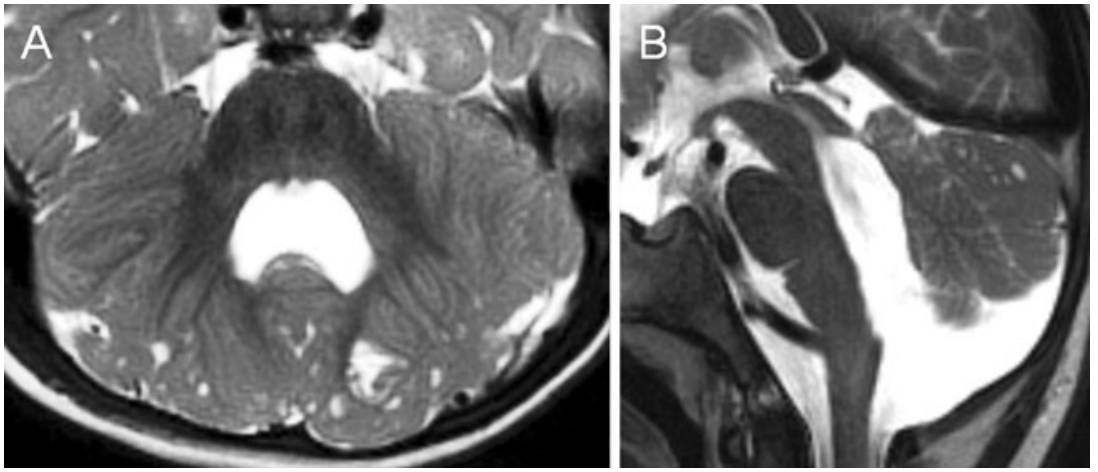

Fig. 2 Axial (a) and midsagittal (b) T2-weighted MR images of patient 3 at the age of 1.8 years show multiple cortical/subcortical cysts located within the cerebellar vermis (mostly anterior and superior part) and both cerebellar hemispheres (mostly posterior and superior parts). Additional abnormalities illustrated: hypoplasia of the inferior part of the cerebellar vermis (b), bilateral cerebellar dysplasia (a), an enlarged fourth ventricle with a peculiar elongated and squared shape (b), and an elongated midbrain (b) 
Fig. 3 Midsagittal (a), axial (b, c), and coronal (d) T2-weighted MR images of patient 4 at the age of 10.5 years show multiple cortical/subcortical cysts located within the cerebellar vermis (mostly anterior and superior part) and both cerebellar hemispheres (mostly posterior and superior parts). Additional abnormalities illustrated: global hypoplasia of the cerebellar vermis (a), bilateral cerebellar dysplasia (b-d), an enlarged fourth ventricle with a peculiar elongated and squared shape (a), and a prominent interpeduncular fossa (b)
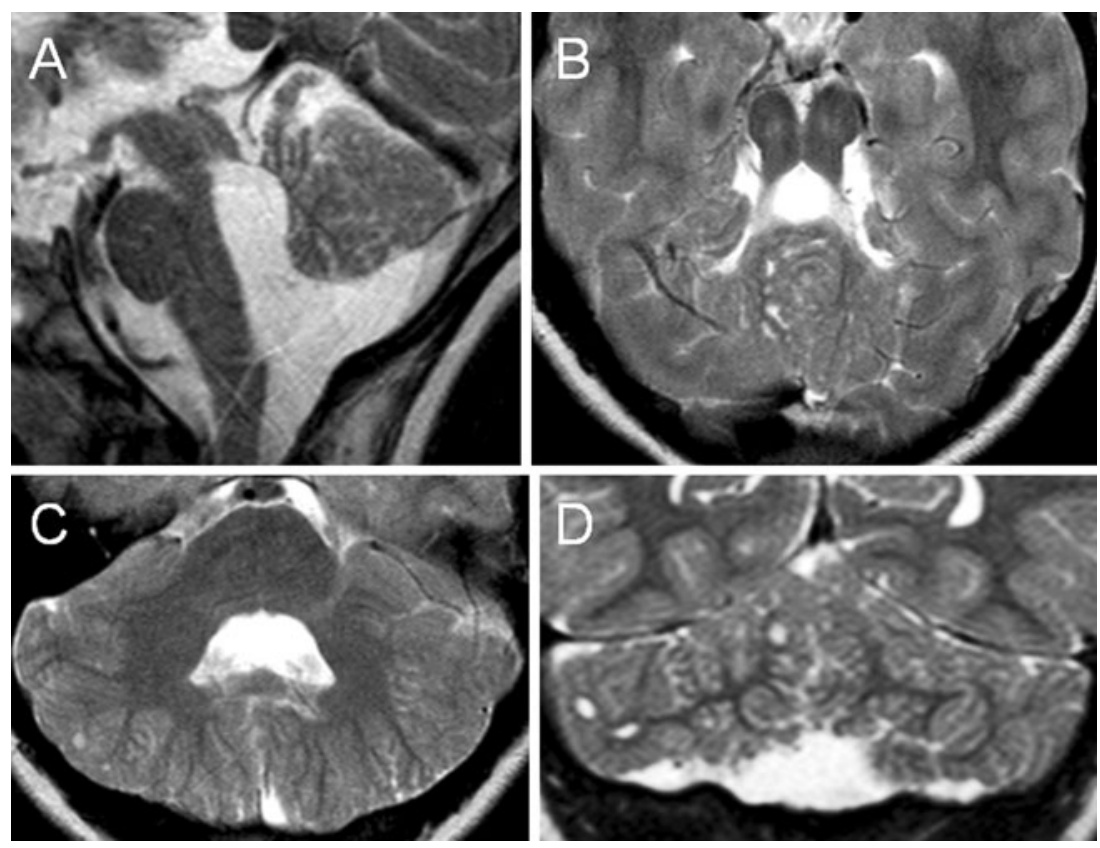

not been reported so far in dystroglycanopathies and GPR56related encephalopathy.

\section{Neuroimaging Phenotype}

Cerebellar cysts are a rare finding in pediatric neuroimaging. They are relatively specific for dystroglycanopathies [2], although they have been occasionally reported in Aicardi syndrome $[13,14]$ and exceptionally in pontocerebellar hypoplasia type 6 [15]. These disorders were excluded in our patients based on history, clinical and neuroimaging findings. Additionally, cerebellar cysts with cerebellar dysplasia, cerebellar hypoplasia, and brain stem abnormalities have been reported in children with GPR56 mutations [12]. However, patients with GPR56 mutations have bilateral fronto-parietal polymicrogyria in an anteroposterior distribution and bilateral patchy white matter changes, which were absent in our children [12]. Genetic analysis did not reveal mutations in GPR56 in all our patients.

Brain involvement in dystroglycanopathies often includes morphological defects of the cerebellum, such as hypoplasia,
Fig. 4 Midsagittal (a), coronal (b), and axial (c, d) T2-weighted MR images of patient 6 at the age of 4.3 years show multiple cortical/subcortical cysts located within the cerebellar vermis (mostly posterior and superior part) and both (right more than left) cerebellar hemispheres (mostly posterior and superior parts). Additional abnormalities illustrated: global hypoplasia of the cerebellar vermis (a), bilateral cerebellar dysplasia (b-d), an enlarged fourth ventricle with a peculiar elongated and squared shape (a), and an elongated midbrain (a)
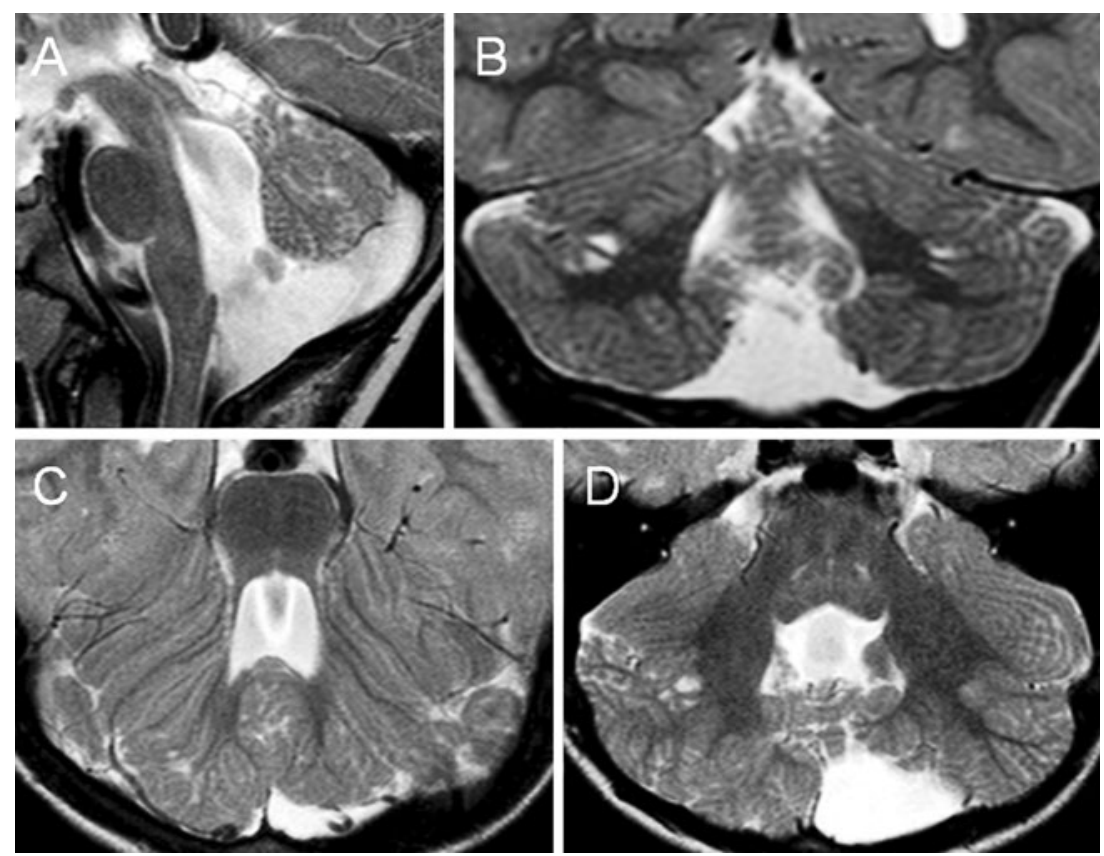
Fig. 5 Midsagittal (a), coronal (b), and axial (c, d) T2-weighted MR images of patient 7 at the age of 11 months show multiple cortical/subcortical cysts located within the cerebellar vermis (mostly anterior and superior part) and both cerebellar hemispheres (mostly posterior and superior parts). Additional abnormalities illustrated: hypoplasia of the inferior part of the cerebellar vermis (a), bilateral cerebellar dysplasia (b-d), an enlarged fourth ventricle with a peculiar elongated and squared shape (a), an elongated midbrain (a), and a short pons (a)
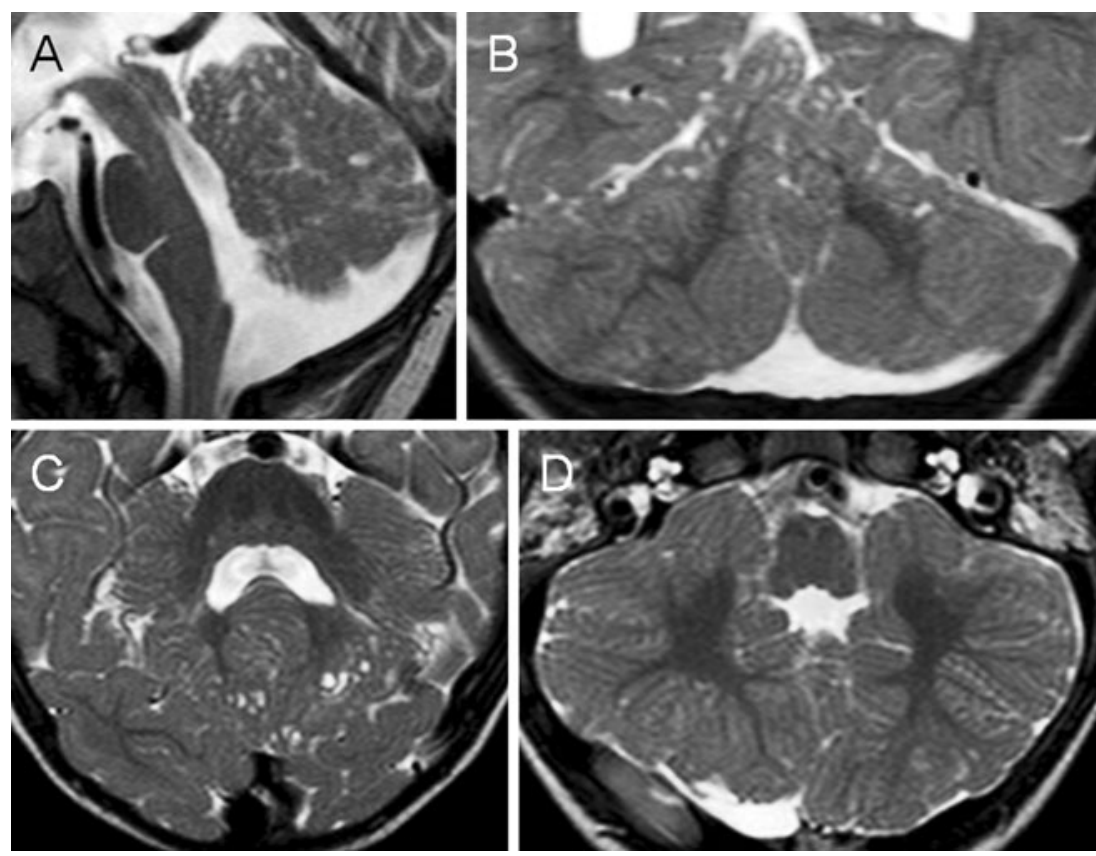

dysplasia, and cystic lesions [2, 5, 28-31]. Neuropathological examinations revealed that cerebellar cysts in CMD are lined by leptomeningeal tissue. They are most likely formed from the subarachnoid spaces that were engulfed by the dysplastic cerebellar folia, particularly in the boundary between normal and dysplastic cerebellar cortex $[32,33]$. In all our patients, cerebellar cysts were associated with cerebellar dysplasia and were located within the most dysplastic cerebellar tissue (antero-superior part

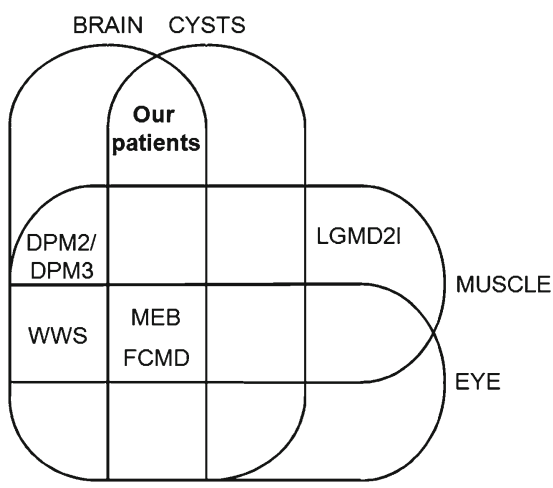

Fig. 6 Venn diagram with schematic distribution of organ involvement and presence of cerebellar cysts for selected $\alpha$-dystroglycanopathies phenotypes. Brain involvement includes clinical (e.g., intellectual disability and seizures) and morphological (e.g., migrational abnormalities, white matter changes, and ventriculomegaly) findings; muscle involvement represents weakness; eye involvement includes, e.g., microphthalmia, retinal dysplasia, glaucoma, and congenital cataracts. $D P M 2 / 3$ dolichol-phosphate mannosyltransferase 2/3, FCMD Fukuyama congenital muscular dystrophy, LGMD2I limb-girdle muscular dystrophy type 2I, $M E B$ muscle-eye-brain disease, $W W S$ Walker-Warburg syndrome of the vermis and postero-superior part of the hemispheres). Cerebellar cysts are characteristic for dystroglycanopathies and have been mostly described in patients with mutations within the FKRP, POMT2, POMGnT1, and LARGE genes [2].

In dystroglycanopathies, involvement of the cerebellum may be isolated or variably associated with morphological abnormalities of the brain stem and supratentorial structures $[2,5]$. Mercuri and colleagues reported on 15 children with CMD due to impaired glycosylation of $\alpha$-dystroglycan and isolated cerebellar involvement [5]. All these children had clinical muscle involvement (weakness) and markedly increased CK (at least 5-fold higher than normal values). In their study on neuroimaging findings in dystroglycanopathies, Clement and coworkers included two children with cerebellar cysts and pontine abnormalities but no obvious cortical changes [2]. However, both patients had ventriculomegaly and supratentorial white matter changes. No data about clinical muscle involvement are reported. Finally, Messina and colleagues also reported on four children with CMD and isolated cerebellar involvement [34]. All these patients had weakness, difficulty in swallowing and markedly increased CK. Our patients did not have supratentorial involvement and only three of them showed subclinical muscle involvement as minimally increased $\mathrm{CK}$.

Morphological brain stem anomalies in dystroglycanopathies generally include pontine hypoplasia (different degree of severity ranging from mild hypoplasia to flat pons such as in MEB or WWS), fused colliculi, ventral pontine cleft in MEB, and pontomesencephalic kinking in WWS [2, 9, 35]. These severe brain stem abnormalities were not present in any of our patients. The 
majority of them had less severe brain stem anomalies including midbrain elongation and/or pontine shortness.

Supratentorial involvement characterizes the most severe phenotypes of dystroglycanopathies such as WWS, MEB, and FCMD [2, 5, 35]. Supratentorial involvement has a wide spectrum of severity and ranges from mild ventriculomegaly, diffuse periventricular white matter changes, and focal areas of polymicrogyria to severe hydrocephalus, generalized white matter changes and diffuse cortical structural abnormalities including cobblestone lissencephaly and an almost absent cortical mantle [2, 5, 9, 35]. Supratentorial involvement in dystroglycanopathies is not consistent and occurs in 40-85\% of the patients $[2,5]$. In our patients, only one had a mild ventriculomegaly as the only supratentorial involvement.

\section{Genotype}

Mutations in 15 genes have been shown to cause a dystroglycanopathy phenotype, including POMT1, POMT2, POMGnT1, FKTN, FKRP, LARGE, ISPD, B3GALNT2, DPM2, DPM3, GTDC2, B3GNT1, TMEM5, SGK196, and $G M P P B$ [4, 9-11, 36-41]. We excluded mutations within the first eight genes in all our patients, including all those genes that, when mutated, have been so far related to cerebellar cysts in neuroimaging. Mutations within the DPM2 and DPM3 genes have been reported to cause a phenotype characterized by intractable seizures, progressive microcephaly, and markedly increased CK $[4,36,37]$. Mutations in GTDC2, TMEM5, and SGK196 have been reported to cause WWS [10]. The phenotype of our patients was not characterized by seizures, progressive microcephaly, markedly increased CK or WWS. One child harboring missense variants in B3GNT1 had cysts within the dysplastic cerebellum but the brain malformation spectrum was wider and included focal cobblestone lissencephaly of the occipital cortex and hyperintense signal of the subjacent white matter [40]. In some of the patients with mutations in $G M P P B$, cerebellar and brain stem malformations have been reported [41]. In children with mutations in $G M P P B$, however, cerebellar cysts have not been found [38, 41]. Therefore, mutations in the DPM2, DPM3, GTDC2, TMEM5, SGK196, B3GNT1, and GMPPB genes are very unlikely in our patients, although not formally excluded. Only in about half of the dystroglycanopathy patients, a mutation was found in the known genes [3-5]. Mutations in GPR56 and $\angle A M B 1$ are related to a cerebral malformation similar to CMD, although not being a dystroglycanopathy itself $[10,38]$. Mutations in GPR56 have been formally excluded in all our patients. Mutations in $L A M B 1$ have been recently shown to cause a constellation of brain malformations including cortical gyral and white matter signal abnormalities, severe cerebellar dysplasia, brain stem hypoplasia, and occipital encephalocele in four children with only mild ocular and muscular abnormal findings [38]. In children with mutations in $L A M B 1$, however, cerebellar cysts have not been found.

\section{Limitations}

We are aware of some limitations in our study: the number of patients is rather small, a formal, quantitative neurocognitive evaluation was only performed in a minority of the patients, and mutations within the newest teammates in $\alpha$-dystroglycanopathies were not formally excluded by direct genetic analysis although cerebellar cysts have not been reported in patients mutated in those genes.

\section{Conclusions}

We report on seven children with the same clinical and imaging phenotype characterized by (1) brain involvement as a cerebellar syndrome with ataxia, OMA and intellectual disability and (2) cerebellar cysts and cerebellar dysplasia. This combination of clinical and neuroimaging findings is reminiscent of $\alpha$-dystroglycanopathies. However, typical features of $\alpha$-dystroglycanopathies, such as clinical muscle involvement and eye involvement were not or only inconsistently present in our patients. Additionally, mutations in the most suitable $\alpha$ dystroglycanopathies genes were not found in our patients. Other well-defined disorders that are phenotypically partially matching with our patients were excluded based on the clinical and/or neuroimaging phenotype or normal genotype. We conclude that the peculiar combination of clinical and neuroimaging findings in our patients suggests that this phenotype may represent a novel syndromic entity, perhaps within the ever-growing dystroglycanopathy spectrum (Fig. 6).

Acknowledgments The authors thank the patients and their families for their cooperation.

Grant/Financial Support This work was partly supported by the Anna Müller Grocholski Foundation, Zurich, Switzerland (grant to AP), the European Research Council (ERC starting grant \#260888 to EMV), and the Italian Ministry of Health (Ricerca Corrente 2013, Ricerca Finalizzata Malattie Rare 2008 to EMV).

Disclosure All coauthors do not report conflicts of interest.

\section{References}

1. Poretti A, Klein A, Boltshauser E. Cerebellar cysts and neuroimaging in congenital muscular dystrophies. In: Boltshauser E, Schmahmann JD, editors. Cerebellar disorders in children. London: Mac Keith Press; 2012. p. 177-83.

2. Clement E, Mercuri E, Godfrey C, et al. Brain involvement in muscular dystrophies with defective dystroglycan glycosylation. Ann Neurol. 2008;64:573-82.

3. Godfrey C, Clement E, Mein R, et al. Refining genotype phenotype correlations in muscular dystrophies with defective glycosylation of dystroglycan. Brain. 2007;130:2725-35. 
4. Mercuri E, Muntoni F. The ever-expanding spectrum of congenital muscular dystrophies. Ann Neurol. 2012;72:9-17.

5. Mercuri E, Messina S, Bruno C, et al. Congenital muscular dystrophies with defective glycosylation of dystroglycan: a population study. Neurology. 2009;72:1802-9.

6. Devisme L, Bouchet C, Gonzales M, et al. Cobblestone lissencephaly: neuropathological subtypes and correlations with genes of dystroglycanopathies. Brain. 2012;135:469-82.

7. Brockington M, Yuva Y, Prandini P, et al. Mutations in the fukutinrelated protein gene (FKRP) identify limb girdle muscular dystrophy 2I as a milder allelic variant of congenital muscular dystrophy MDC1C. Hum Mol Genet. 2001;10:2851-9.

8. Roscioli T, Kamsteeg EJ, Buysse K, et al. Mutations in ISPD cause Walker-Warburg syndrome and defective glycosylation of alphadystroglycan. Nat Genet. 2012;44:581-5.

9. Willer T, Lee H, Lommel M, et al. ISPD loss-of-function mutations disrupt dystroglycan O-mannosylation and cause Walker-Warburg syndrome. Nat Genet. 2012;44:575-80.

10. Manzini MC, Tambunan DE, Hill RS, et al. Exome sequencing and functional validation in zebrafish identify GTDC2 mutations as a cause of Walker-Warburg syndrome. Am J Hum Genet. 2012;91:541-7.

11. Jae LT, Raaben M, Riemersma M, et al. Deciphering the glycosylome of dystroglycanopathies using haploid screens for lassa virus entry. Science. 2013;340:479-83.

12. Bahi-Buisson N, Poirier K, Boddaert N, et al. GPR56-related bilateral frontoparietal polymicrogyria: further evidence for an overlap with the cobblestone complex. Brain. 2010;133:3194-209.

13. Hopkins B, Sutton VR, Lewis RA, Van den Veyver I, Clark G. Neuroimaging aspects of Aicardi syndrome. Am J Med Genet A. 2008;146A:2871-8.

14. Steffensen TS, Gilbert-Barness E, Lacson A, Margo CE. Cerebellar migration defects in Aicardi syndrome: an extension of the neuropathological spectrum. Fetal Pediatr Pathol. 2009;28:24-38.

15. Glamuzina E, Brown R, Hogarth K, et al. Further delineation of pontocerebellar hypoplasia type 6 due to mutations in the gene encoding mitochondrial arginyl-tRNA synthetase, RARS2. J Inherit Metab Dis. 2012;35:459-67.

16. Doherty D, Millen KJ, Barkovich AJ. Midbrain and hindbrain malformations: advances in clinical diagnosis, imaging, and genetics. Lancet Neurol. 2013;12:381-93.

17. Shevell M. Global developmental delay and mental retardation or intellectual disability: conceptualization, evaluation, and etiology. Pediatr Clin N Am. 2008;55:1071-84.

18. Boltshauser E, Poretti A. Nonprogressive congenital ataxia. In: Boltshauser E, Schmahmann JD, editors. Cerebellar disorders in children. London: Mac Keith Press; 2012. p. 135-9.

19. Poretti A, Boltshauser E. Congenital ataxia (Table 1). In: Boltshauser E, Schmahmann JD, editors. Cerebellar disorders in children. London: Mac Keith Press; 2012. p. 399-400.

20. Poretti A, Boltshauser E. Ataxia and ocular motor apraxia (Table 7). In: Boltshauser E, Schmahmann JD, editors. Cerebellar disorders in children. London: Mac Keith Press; 2012. p. 406.

21. Romani M, Micalizzi A, Valente EM. Joubert syndrome: congenital cerebellar ataxia with the molar tooth. Lancet Neurol. 2013;12:894-905.

22. Kondo A, Saito Y, Floricel F, Maegaki Y, Ohno K. Congenital ocular motor apraxia: clinical and neuroradiological findings, and long-term intellectual prognosis. Brain Dev. 2007;29:431-8.

23. Sargent MA, Poskitt KJ, Jan JE. Congenital ocular motor apraxia: imaging findings. AJNR Am J Neuroradiol. 1997;18:1915-22.
24. Poretti A, Huisman TA, Scheer I, Boltshauser E. Joubert syndrome and related disorders: spectrum of neuroimaging findings in 75 patients. AJNR Am J Neuroradiol. 2011;32:1459-63.

25. Anheim M, Tranchant C, Koenig M. The autosomal recessive cerebellar ataxias. N Engl J Med. 2012;366:636-46.

26. Cirak S, Foley AR, Herrmann R, et al. ISPD gene mutations are a common cause of congenital and limb-girdle muscular dystrophies. Brain. 2013;136:269-81.

27. Quattrocchi CC, Zanni G, Napolitano A, et al. Conventional magnetic resonance imaging and diffusion tensor imaging studies in children with novel GPR56 mutations: further delineation of a cobblestone-like phenotype. Neurogenetics. 2013;14:77-83.

28. Louhichi N, Triki C, Quijano-Roy S, et al. New FKRP mutations causing congenital muscular dystrophy associated with mental retardation and central nervous system abnormalities. Identification of a founder mutation in Tunisian families. Neurogenetics. 2004;5: $27-34$.

29. Topaloglu H, Brockington M, Yuva Y, et al. FKRP gene mutations cause congenital muscular dystrophy, mental retardation, and cerebellar cysts. Neurology. 2003;60:988-92.

30. Mercuri E, Topaloglu H, Brockington M, et al. Spectrum of brain changes in patients with congenital muscular dystrophy and FKRP gene mutations. Arch Neurol. 2006;63:251-7.

31. Yis U, Uyanik G, Heck PB, et al. Fukutin mutations in non-Japanese patients with congenital muscular dystrophy: less severe mutations predominate in patients with a non-Walker-Warburg phenotype. Neuromuscul Disord. 2011;21:20-30.

32. Aida N, Yagishita A, Takada K, Katsumata Y. Cerebellar MR in Fukuyama congenital muscular dystrophy: polymicrogyria with cystic lesions. AJNR Am J Neuroradiol. 1994;15:1755-9.

33. Aida N, Tamagawa K, Takada K, et al. Brain MR in Fukuyama congenital muscular dystrophy. AJNR Am J Neuroradiol. 1996;17: 605-13.

34. Messina S, Tortorella G, Concolino D, et al. Congenital muscular dystrophy with defective alpha-dystroglycan, cerebellar hypoplasia, and epilepsy. Neurology. 2009;73:1599-601.

35. Barkovich AJ. Neuroimaging manifestations and classification of congenital muscular dystrophies. AJNR Am J Neuroradiol. 1998;19:1389-96.

36. Lefeber DJ, Schonberger J, Morava E, et al. Deficiency of Dol-PMan synthase subunit DPM3 bridges the congenital disorders of glycosylation with the dystroglycanopathies. Am J Hum Genet. 2009;85:76-86.

37. Barone R, Aiello C, Race V, et al. DPM2-CDG: a muscular dystrophy-dystroglycanopathy syndrome with severe epilepsy. Ann Neurol. 2012;72:550-8.

38. Radmanesh F, Caglayan AO, Silhavy JL, et al. Mutations in LAMB1 cause cobblestone brain malformation without muscular or ocular abnormalities. Am J Hum Genet. 2013;92:468-74.

39. Stevens E, Carss KJ, Cirak S, et al. Mutations in B3GALNT2 cause congenital muscular dystrophy and hypoglycosylation of alphadystroglycan. Am J Hum Genet. 2013;92:354-65.

40. Buysse K, Riemersma M, Powell G, et al. Missense mutations in beta-1,3- $N$-acetylglucosaminyltransferase 1 (B3GNT1) cause Walker-Warburg syndrome. Hum Mol Genet. 2013;22:1746-54.

41. Carss KJ, Stevens E, Foley AR, et al. Mutations in GDP-mannose pyrophosphorylase $\mathrm{b}$ cause congenital and limb-girdle muscular dystrophies associated with hypoglycosylation of alpha-dystroglycan. Am J Hum Genet. 2013;93:29-41. 